\title{
WEIGHTED ITERATED HARDY-TYPE INEQUALITIES
}

\author{
Amiran Gogatishvili and Rza CH. Mustafayev
}

\begin{abstract}
In this paper reduction and equivalence theorems for the boundedness of the composition of a quasilinear operator $T$ with the Hardy and Copson operators in weighted Lebesgue spaces are proved. New equivalence theorems are obtained for the operator $T$ to be bounded in weighted Lebesgue spaces restricted to the cones of monotone functions, which allow to change the cone of non-decreasing functions to the cone of non-increasing functions and vice versa not changing the operator $T$. New characterizations of the weighted Hardy-type inequalities on the cones of monotone functions are given. The validity of so-called weighted iterated Hardy-type inequalities are characterized.
\end{abstract}

Mathematics subject classification (2010): 26D10, 26D15.

Keywords and phrases: Quasilinear operators, iterated Hardy inequalities, weights.

\section{REFERENCES}

[1] M. I. Aguilar Cañestro, P. Ortega Salvador, C. Ramirez Torreblanca, Weighted bilinear Hardy inequalities, J. Math. Anal. Appl. 387 (2012), no. 1, 320-334, DOI 10.1016/j.jmaa.2011.08.078, MR2845753.

[2] K. F. ANDERSEN, Weighted inequalities for the Stieltjes transformation and Hilbert's double series, Proc. Roy. Soc. Edinburgh Sect. A, 86 (1980), no. 1-2, 75-84, DOI 10.1017/S0308210500012014, MR580247 (82b:26020).

[3] M. A. Ariño, B. Muckenhoupt, Maximal functions on classical Lorentz spaces and Hardy's inequality with weights for nonincreasing functions, Trans. Amer. Math. Soc. 320 (1990), no. 2, 727 735, DOI 10.2307/2001699, MR989570 (90k:42034).

[4] G. Bennett, K.-G. Grosse-Erdmann, Weighted Hardy inequalities for decreasing sequences and functions, Math. Ann. 334 (2006), no. 3, 489-531, DOI 10.1007/s00208-005-0678-7, MR2207873 (2006m:26038)

[5] D. W. BoyD, The Hilbert transform on rearrangement-invariant spaces, Canad. J. Math. 19 (1967), 599-616, MR0212512 (35 \#3383).

[6] V. I. Burenkov, A. Gogatishvili, V. S. Guliyev, R. Ch. Mustafayev, Boundedness of the fractional maximal operator in local Morrey-type spaces, Complex Var. Elliptic Equ. 55 (2010), no. 8-10, 739-758, MR2674862 (2011f:42015).

[7] V. I. Burenkov, A. Gogatishvili, V. S. Guliyev, R. Ch. Mustafayev, Boundedness of the Riesz potential in local Morrey-type spaces, Potential Anal. 35 (2011), no. 1, 67-87, MR2804553 (2012d:42027).

[8] V. I. BuRENKov, M. L. Goldman, Calculation of the norm of a positive operator on the cone of monotone functions, Trudy Mat. Inst. Steklov. 210 (1995), no. Teor. Funktsii i Differ. Uravn., 65-89 (Russian). In honor of the ninetieth birthday of Academician S. M. Nikolskii (Russian), MR1421378 (97m:47038).

[9] V. I. BURENKov, R. Oinarov, Necessary and sufficient conditions for boundedness of the Hardytype operator from a weighted Lebesgue space to a Morrey-type space, Math. Inequal. Appl. 16 (2013), no. 1, 1-19, MR3060376.

[10] M. Carro, A. Gogatishvili, J. Martin, L. Pick, Weighted inequalities involving two Hardy operators with applications to embeddings of function spaces, J. Operator Theory 59 (2008), no. 2, 309-332, MR2411048 (2009f:26024). 
[11] M. Carro, L. Pick, J. Soria, V. D. Stepanov, On embeddings between classical Lorentz spaces, Math. Inequal. Appl. 4 (2001), no. 3, 397-428, DOI 10.7153/mia-04-37, MR1841071 (2002d:46026).

[12] J. M. CARro, J. Soria, Boundedness of some integral operators, Canad. J. Math. 45 (1993), no. 6, 1155-1166, DOI 10.4153/CJM-1993-064-2, MR1247539 (95d:47064).

[13] W. D. Evans, A. Gogatishvili, B. Opic, The $\rho$-quasiconcave functions and weighted inequalities, Inequalities and applications, Internat. Ser. Numer. Math. vol. 157, Birkhäuser, Basel, 2009, pp. 121-132, MR2758974 (2012a:26025).

[14] A. Gogatishvili, Discretization and anti-discretization of function spaces, In the proceedings of the The Autumn Conference Mathematical Society of Japan, September 25-28, Shimane University, Matsue (2002), 63-72.

[15] A. Gogatishvili, M. Johansson, C. A. Okpoti, L.-E. Persson, Characterisation of embeddings in Lorentz spaces, Bull. Austral. Math. Soc. 76 (2007), no. 1, 69-92, DOI 10.1017/S0004972700039484, MR2343440 (2008j:46017).

[16] A. Gogatishvili, A. Kufner, L.-E. Persson, Some new scales of weight characterizations of the class $B_{p}$, Acta Math. Hungar. 123 (2009), no. 4, 365-377, DOI 10.1007/s10474-009-8132-z, MR2506756 (2010b:42021).

[17] A. Gogatishvili, A. Kufner, L.-E. Persson, The weighted Stieltjes inequality and applications, Math. Nachr. 286 (2013), no. 7, 659-668, MR3060837.

[18] A. Gogatishvili, R. Ch. Mustafayev, L.-E. Persson, Some new iterated Hardy-type inequalities, J. Funct. Spaces Appl., (2012), Art. ID 734194, 30, MR3000818.

[19] A. Gogatishvili, R. Ch. Mustafayev, L.-E. Persson, Some new iterated Hardy-type inequalities: the case $\theta=1$, J. Inequal. Appl. (2013), 29 pp., DOI 10.1186/1029-242X-2013-515.

[20] A. Gogatishvili, L.-E. Persson, V. D. Stepanov, P. Wall, On scales of equivalent conditions that characterize the weighted Stieltjes inequality, Dokl. Akad. Nauk 447 (2012), no. 1, 13-14, (Russian). Dokl. Math. 86 (2012), no. 3, 738-739, MR3075082.

[21] A. Gogatishvili, L.-E. Persson, V. D. Stepanov, P. Wall, Some scales of equivalent conditions to characterize the Stieltjes inequality: the case $q<p$, Math. Nachr. 287 (2014), no. 2-3, 242-253, DOI 10.1002/mana.201200118, MR3163577.

[22] A. Gogatishvili, L. Pick, Duality principles and reduction theorems, Math. Inequal. Appl. 3 (2000), no. 4, 539-558, MR1786395 (2002c:46056),

[23] A. Gogatishvili, L. Pick, A reduction theorem for supremum operators, J. Comput. Appl. Math. 208 (2007), no. 1, 270-279, MR2347749 (2009a:26013).

[24] A. Gogatishvili, V. D. Stepanov, Integral operators on cones of monotone functions, Dokl. Akad. Nauk 446 (2012), no. 4, 367-370, (Russian), English transl., Dokl. Math. 86 (2012), no. 2, 650-653, DOI 10.1134/S1064562412050158, MR3053208.

[25] A. Gogatishvili, V. D. Stepanov, Operators on cones of monotone functions, Dokl. Akad. Nauk 445 (2012), no. 6, 618-621, (Russian), English transl., Dokl. Math. 86 (2012), no. 1, 562-565, MR3050526.

[26] A. Gogatishvili, V. D. Stepanov, Reduction theorems for operators on the cones of monotone functions, J. Math. Anal. Appl. 405 (2013), no. 1, 156-172, DOI 10.1016/j.jmaa.2013.03.046, MR3053495.

[27] A. Gogatishvili, V. D. Stepanov, Reduction theorems for weighted integral inequalities on the cone of monotone functions, Uspekhi Mat. Nauk 68 (2013), no. 4 (412), 3-68 (Russian, with Russian summary); English transl., Russian Math. Surveys 68 (2013), no. 4, 597-664, MR3154814.

[28] M. L. Goldman, Sharp estimates for the norms of Hardy-type operators on cones of quasimonotone functions, Tr. Mat. Inst. Steklova 232 (2001), no. Funkts. Prostran., Garmon. Anal., Differ. Uravn., 115-143 (Russian, with Russian summary); English transl., Proc. Steklov Inst. Math. 1 (232) (2001), 109-137, MR1851444 (2002m:42019).

[29] M. L. Goldman, Order-sharp estimates for Hardy-type operators on the cones of functions with properties of monotonicity, Eurasian Math. J. 3 (2012), no. 2, 53-84, MR3024120.

[30] M. L. Goldman, Order-sharp estimates for Hardy-type operators on cones of quasimonotone functions, Eurasian Math. J. 2 (2011), no. 3, 143-146, MR2910846.

[31] H. P. Heinig, V. D. Stepanov, Weighted Hardy inequalities for increasing functions, Canad. J. Math. 45 (1993), no. 1, 104-116, DOI 10.4153/CJM-1993-006-3, MR1200323 (93j:26011). 
[32] M. Johansson, V. D. STEPANov, E. P. UshaKova, Hardy inequality with three measures on monotone functions, Math. Inequal. Appl. 11 (2008), no. 3, 393-413, DOI 10.7153/mia-11-30, MR2431205 (2010d:26024).

[33] A. Kufner, L.-E. Persson, Weighted inequalities of Hardy type, World Scientific Publishing Co., Inc., River Edge, NJ, 2003, MR1982932 (2004c:42034).

[34] A. Kufner, L. Maligranda, L.-E. Persson, The Hardy inequality, Vydavatelský Servis, Plzeň 162, 2007, About its history and some related results, MR2351524 (2008j:26001).

[35] S. LAI, Weighted norm inequalities for general operators on monotone functions, Trans. Amer. Math. Soc. 340 (1993), no. 2, 811-836, DOI 10.2307/2154678, MR1132877 (94b:42005).

[36] B. Opic, A. Kufner, Hardy-type inequalities, Pitman Research Notes in Mathematics Series 219, Longman Scientific \& Technical, Harlow, 1990, MR1069756 (92b:26028).

[37] O. V. Popova, Hardy-type inequalities on cones of monotone functions, Sibirsk. Mat. Zh. 53 (2012), no. 1, 187-204, (Russian, with Russian summary); English transl., Sib. Math. J. 53 (2012), no. 1, 152-167, MR2962198.

[38] D. V. Prokhorov, V. D. Stepanov, On weighted Hardy inequalities in mixed norms, Proc. Steklov Inst. Math. 283 (2013), 149-164.

[39] D. V. Prokhorov, V. D. Stepanov, Weighted estimates for a class of sublinear operators, Dokl. Akad. Nauk 453 (2013), no. 5, 486-488 (Russian); English transl., Dokl. Math. 88 (2013), no. 3, 721-723, MR3203323.

[40] E. SAWYER, Boundedness of classical operators on classical Lorentz spaces, Studia Math. 96 (1990), no. 2, 145-158, MR1052631 (91d:26026).

[41] G. Sinnamon, A note on the Stieltjes transformation, Proc. Roy. Soc. Edinburgh Sect. A 110 (1988), no. 1-2, 73-78, DOI 10.1017/S0308210500024860, MR963841 (90a:26026).

[42] G. Sinnamon, Embeddings of concave functions and duals of Lorentz spaces, Publ. Mat. 46 (2002), no. 2, 489-515, MR1934367 (2003h:46042).

[43] G. Sinnamon, Transferring monotonicity in weighted norm inequalities, Collect. Math. 54 (2003), no. 2, 181-216, MR1995140 (2004m:26031).

[44] G. Sinnamon, Hardy's inequality and monotonicity, in: Proc. "Function Spaces, Differential operators and Nonlinear Analysis" (FSDONA 2004), Acad. Sci., Czech Republic, Milovy, (2004), 292-310.

[45] G. Sinnamon, V. D. Stepanov, The weighted Hardy inequality: new proofs and the case $p=1$, J. London Math. Soc. (2) 54 (1996), no. 1, 89-101, DOI 10.1112/jlms/54.1.89, MR1395069 (97e:26021).

[46] V. D. StePanov, The weighted Hardy's inequality for nonincreasing functions, Trans. Amer. Math. Soc. 338 (1993), no. 1, 173-186, DOI 10.2307/2154450, MR1097171 (93j:26012).

[47] V. D. STEPANOV, Integral operators on the cone of monotone functions, J. London Math. Soc. (2) 48 (1993), no. 3, 465-487, DOI 10.1112/jlms/s2-48.3.465, MR1241782 (94m:26025). 